\title{
Elasticity model of a supercoiled DNA molecule
}

\author{
C. Bouchiat and M. Mézard \\ Laboratoire de Physique Théorique de l'Ecole Normale Supérieurt \\ 24 rue Lhomond, 75231 Paris Cedex 05, France
}

\begin{abstract}
Within a simple elastic theory, we study the elongation versus force characteristics of a supercoiled DNA molecule at thermal equilibrium in the regime of small supercoiling. The partition function is mapped to the path integral representation for a quantum charged particle in the field of a magnetic monopole with unquantized charge. We show that the theory is singular in the continuum limit and must be regularised at an intermediate length scale. We find good agreement with existing experimental data, and point out how to measure the twist rigidity accurately. LPTENS 97/28.
\end{abstract}

The measurements on single DNA molecules, beside their possible biological interest, provide a wonderful laboratory for the physical studies of a single polymer chain. For instance, recent experiments have shown that the elongation versus force characteristics of a single DNA molecule [2] is very well fitted [3] by the well known worm-like chain (WLC) [4 which describes a chain by an elastic continuous curve at thermal equilibrium, with a single elastic constant, the persistence length $A$ characterizing the bending energy. The WLC can be solved analytically by mapping it to a quantum mechanical problem. Its partition function is nothing but a Euclidean path integral for a quantum dumbbell, which can be computed, in the relevant limit of long chains, by finding the ground state of the corresponding Hamiltonian.

Our work is motivated by the more recent experiments which have measured the elongation versus force characteristics of a supercoiled DNA molecule [5]. We use the simplest generalization of the WLC with twist rigidity, which is supposed to work at small supercoiling angles. The DNA molecule is described by a thin elastic rod involving a new elastic constant, the twist rigidity $C$. The stress imposed by twisting the endpoint of an open rod can be absorbed both in some twist (if the axis of the rod is a straight line), and in some deformation of the rod's axis. This decomposition is well known in the case of closed rods, for which the experimental constraint is a topological invariant [6]. It results in a subtle competition involving the creation of plectonemes which has received quite a lot of attention, both for the study of the ground state, and also taking care phenomenologically of thermal fluctuations around some low energy configurations [7, 9, 8 . In contrast, we keep here to the simplest regime of small supercoiling, but we provide a full analytic and numerical study of the twisted open rod at thermal equilibrium at a finite temperature, extending thus the standard WLC analysis to this case. While it has been known for some time that the elastic thin rod model agrees qualitatively with experimental results on DNA, this knowledge was based only on some Monte Carlo simulations 12,13. . Our analytic study of the worm-like rod chain (WLRC) shows very good quantitative agreement with the experimental data at small supercoiling and al- lows for a precise determination of the ratio $C / A$, which was poorly known so far.

We shall show that beside the two elastic constants describing the bending and twisting rigidity, one needs to introduce an intermediate lengthscale $b$ between the microscopic interbasepair distance and the persistence length, which plays the role of a short distance cutoff. Obviously the rod description cannot hold below a cutoff length scale $b$ which is at least of the order of the double helix pitch $p \simeq 0.34 \mathrm{~nm}$, since our description averages over these periodic oscillations. As we shall see the WLRC continuous limit is singular and shows properties qualitatively very different from any discretized version of the chain. So the existence of a cutoff, which is expected on physical ground, is crucial, but the final results turn out to be fairly independent of its precise value, within a reasonable range around $.5 \mathrm{~nm}$. Similar singularities of the continuous limit are well known in the winding properties of pure random walks 10]. Their appearance here is not fortuitous since the worm-like rod chain is related to random walks in rotation space. It is interesting that supercoiled DNA presents an experimental system where these subtleties of the continuous limit of random walks turn out to be relevant.

The WLRC, already studied in 12,9,8,13, is described in the continuous limit by the orthonormal triedron $\{\mathbf{t}(s), \mathbf{u}(s), \mathbf{n}(s)\}$ where $s$ is the arc length along the molecule, $\mathbf{t}$ is the unit vector tangent to the chain, and $\mathbf{n}$ describes the orientation of the rod. For describing DNA, this triedron is obtained by applying a rotation $\mathcal{R}(s)$ to a reference triedron which characterizes the natural helical structure of the molecule. The rotation $\mathcal{R}(s)$ is parametrized by the usual three Euler angles $\theta(s), \phi(s), \psi(s)$, and the reference triedron is such that $\theta(s)=0, \phi(s)+\psi(s)=\omega_{0} s$, where $\omega_{0}$ is the rotation per unit length of the base axis in a relaxed rectilinear DNA molecule. With the above definition, the set of $s$ dependent Euler angles $\theta(s), \phi(s), \psi(s)$ describes the general deformations of the DNA molecule with respect to the relaxed rectilinear configuration. We neglect selfavoidance, the energy of a chain of length $L$ is purely elastic: $E_{e l}=\int_{0}^{L} d s\left(e_{b}+e_{t}\right)$, with the bend and twist energy densities given by: 


$$
\begin{aligned}
& e_{b}=\frac{A}{2}\left|\frac{d \mathbf{t}(s)}{d s}\right|^{2}=\frac{A}{2}\left(\dot{\phi}^{2} \sin ^{2} \theta+\dot{\theta}^{2}\right) \\
& e_{t}=\frac{C}{2}\left|\mathbf{t}(s) \times \mathbf{n}(s) \cdot \frac{d \mathbf{n}(s)}{d s}-\omega_{0}\right|^{2}=\frac{C}{2}(\dot{\psi}+\dot{\phi} \cos \theta)^{2}
\end{aligned}
$$

where the dot stands for the $s$ derivative. We work in units where the temperature $k_{B} T=1$, so that $A$ and $C$ have dimension of a length. The discretised version is defined by quantifying $s$ as an integer multiple of an elementary length scale $b$, and approximating integrals and derivatives by sums and differences, while keeping the periodicity. We study the equilibrium properties of such a rod pulled by a force $\mathbf{F}=F \mathbf{z}$. The total energy is thus $E=E_{e l}-F \int_{0}^{L} d s \cos \theta(s)$.

The partition function of the elastic chain described by eq.(1) is nothing but the Euclidean path integral for a quantum symmetric top, with the important difference that the eigenfunctions are not periodic in the angles $\psi$ and $\phi$. Therefore the momenta conjugate to these angles will not be quantized. In our analytical work, we suppose for simplicity that the boundary values of the Euler angles are $\theta(0)=\theta(L)=0$, and we define $\phi(0)=\psi(0)=0$. Then the experimentally imposed supercoiling angle $\chi$ amounts to fixing: $\psi(L)+\phi(L)=\chi$. In the open DNA chain the continuous angle $\chi$ replaces the topological linking number $L_{k}$. We shall limit ourselves to configurations where the Euler angles are regular enough, such that $\chi$ can be written as the integral $\int_{0}^{L} d s(\dot{\psi}+\dot{\phi})$. It is convenient to introduce the rod twist $T_{w}$, given by the integral $T_{w}=\int_{0}^{L} d s(\dot{\psi}+\dot{\phi} \cos \theta) ; T_{w}$ appears as a Gaussian variable in the partition function path integral. We now define a 'local writhe' contribution to the supercoiling angle $\chi_{W}$ as $\chi_{W}=\chi-T_{w}=\int_{0}^{L} d s \dot{\phi}(1-\cos \theta)$. The above decomposition [8] is reminiscent of the one performed in the case of of closed chain where the topological linking number $L_{k}$ is decomposed into twist and writhe [11].

The partition function for a fixed value of $\chi$ is given by the path integral in the space of Euler angles:

$$
Z=\int d[\cos \theta, \phi, \psi] \delta\left(\chi-\int_{0}^{L} d s(\dot{\phi}+\dot{\psi})\right) e^{-E}
$$

After introducing an integral representation of the $\delta$ function which fixes $\chi$, one can perform the gaussian path integral on the angle $\psi$. $Z$ is then expressed as a path integral on the two angles $\cos \theta$ and $\phi$, with an effective energy:

$$
\mathcal{E}=\int_{0}^{L} d s\left(e_{b}-F \cos \theta\right)+\frac{C}{2 L}\left(\chi-\chi_{W}\right)^{2}
$$

This form (3) is useful for numerical simulations [12] after a proper discretization, but not for analytic computation, due to its non local character. Alternatively we can compute the $\chi$ Fourier transform $\tilde{Z}=\int d \chi Z \exp (-i k \chi)$, which is again given by a path integral on the two angles $\cos \theta$ and $\phi$, with the effective energy:

$$
\tilde{\mathcal{E}}(k)=\frac{k^{2} L}{2 C}+\int_{0}^{L} d s\left(e_{b}-F \cos \theta+i k \dot{\phi}(1-\cos \theta)\right)
$$

This last form has an appealing quantum mechanical interpretation: If one analytically continues the s-integral towards the imaginary axis, one recognizes the action integral of a particle with unit charge moving on the unit sphere under the joint action of the electric field $F$ and the magnetic field $A_{\phi}=k(1-\cos \theta)$ of a magnetic monopole of charge $\mathrm{k}$. One easily deduces the corresponding Hamiltonian $H$, by substituting $p_{\phi}=-i \frac{\partial}{\partial \phi}$ by $p_{\phi}-A_{\phi}$ in the WLC Hamiltonian (which corresponds to $A_{\phi}=0$ ). Because of the averaging over the final $\phi=\phi(L)$, only the eigenvalue $m=0$ of $p_{\phi}$ contributes and we can set $p_{\phi}=0$ in $H$. We work with the dimensionless quantities $\hat{H}=H / A$ and $\alpha \equiv A F$, in terms of which we get:

$$
\hat{H}=-\frac{1}{2 \sin \theta} \frac{\partial}{\partial \theta} \sin \theta \frac{\partial}{\partial \theta}-\alpha \cos \theta+\frac{k^{2}}{2} \frac{1-\cos \theta}{1+\cos \theta}
$$

Introducing the eigenstates and the eigenvalues of $\hat{H}$, $\hat{H} \Psi_{n}(k, \theta)=\epsilon_{n}\left(k^{2}, \alpha\right) \Psi_{n}(k, \theta)$, the Fourier transformed partition function $\tilde{Z}$ can be written as the sum:

$$
\tilde{Z}=\sum_{n}\left|\Psi_{n}(k, 0)\right|^{2} \exp \left(-\frac{L}{A}\left(\epsilon_{n}\left(k^{2}, \alpha\right)+\frac{k^{2} A}{2 C}\right)\right)
$$

In the large $L$ limit, the sum over the eigenstates is dominated by the one with lowest energy $\epsilon_{0}\left(\alpha, k^{2}\right)$, if $L / A \gg \Delta \epsilon$ where $\Delta \epsilon$ is the energy gap between the ground state and the nearest excited state of $\hat{H}$. This gives the approximate expression for the partition function $Z$ :

$$
Z \simeq \int d k \exp \left(-\frac{L}{A}\left(\epsilon_{0}\left(k^{2}, \alpha\right)+\frac{k^{2} A}{2 C}\right)+i k \chi\right)
$$

Therefore one can deduce, from the ground state energy $\epsilon_{0}\left(\alpha, k^{2}\right)$ of the Hamiltonian $\hat{H}$, the observable properties of a long WLRC, of which we now discuss two important ones. The relative extension of the chain in the direction of the force is given by $\langle z\rangle / L=(A / L) \frac{\partial \ln Z}{\partial \alpha}$. If instead of constraining $\chi$ one measures its thermal fluctuations, their probability distribution is just $P(\chi) \propto Z$. For instance the second moment is given by:

$$
<\chi^{2}>=\frac{L}{C}+\frac{2 L}{A} \lim _{k^{2} \rightarrow 0} \frac{\partial \epsilon_{0}\left(k^{2}, \alpha\right)}{\partial k^{2}}
$$

This expression shows that the WLRC is pathological because of "giant" fluctuations of $\chi_{W}$. The contribution to $<\chi^{2}>$ from the twist fluctuations, $\frac{L}{C}$, scales 
linearly in $L$, as one expects in a one dimension statistical mechanics system with a finite correlation length. In contrast the second piece of (8) giving the contribution from $\left\langle\chi_{W}^{2}\right\rangle$, is divergent: evaluating $\epsilon_{0}\left(k^{2}, \alpha\right)$ at small $k^{2}$ from standard perturbation theory, we find $\left\langle\chi_{W}{ }^{2}\right\rangle=(L / A)\langle(1-\cos \theta) /(1+\cos \theta)\rangle_{0}$ where \langle\rangle$_{0}$ is the quantum average taken on the groundstate $\Phi_{0}(\theta)$ of the WLC Hamiltonian (which is $\hat{H}$ at $k=0$ ). As $\Phi_{0}(\pi) \neq 0$ (for any finite force), we get a logarithmically divergent result. One can show that $\epsilon\left(\alpha, k^{2}\right) \sim \epsilon(\alpha, 0)+|k| \Phi_{0}(\pi)^{2}$. This linear behaviour of the energy in $|k|$ shows that $P(\chi)$ has a Cauchy tail, and thus a diverging second moment. The Cauchy distribution has been verified in the limit of a vanishing force, $\alpha=0$, where the eigenfunctions can be found exactly in terms of Jacobi polynomials. A related consequence is that the extensive part of the average extension is unchanged by the supercoiling angle $\chi$ at small forces: $\langle z\rangle / L \simeq 2 \alpha / 3$ independently of $F$, in striking contradiction to experiment.

In contrast to the WLC, the continuous limit of the WLRC is singular. This singular behaviour could have been anticipated since $\hat{H}$ describes the motion of a charged particle in a magnetic monopole with an unquantized magnetic charge, a notoriously ill defined problem if no cutoff is provided near $\theta=\pi$. As we have argued previously, the WLRC model is not expected to give a good description of supercoiled DNA unless one introduces the cut-off length scale $b$. The non trivial fact is that this existence of a cutoff affects the 'macroscopic' properties taking place on the length scale of the whole molecule.

In order to validate the discretized WLRC, we have performed a Monte Carlo simulation, mostly using the discretized version of (3). Such simulations are known to account well for the observed behaviour of circular DNA 12], and have been used recently for the study of chains elongated with large supercoiling angles [13. With respect to these works, we have discarded the self avoidance, since our aim is to test the discretized WLRC without self avoidance. We have discretized the chain with elementary rods of length $b=A / 10$, and simulated mostly chains of length $L=30 A$. In order to facilitate the thermalisation, we have relaxed in the simulation the constraint $\theta(L)=0$, which should not affect the extensive quantities. The elementary moves which we used was to scan sequentially each point $i=1, \ldots, N=L / b$ in the chain, and propose a global rotation of the tangent vectors $\mathbf{t}_{j}, j=i, \ldots, N$ around a random axis with an angle $\gamma$ taken with a flat distribution in $\left[-\gamma_{0}, \gamma_{0}\right]$, where $\gamma_{0}$ is chosen such that the acceptance rate of the moves is of order .5. These rotations of a fraction of the chain were the best we found for insuring a relatively fast thermalization 14. The results presented in Fig.1, obtained with $C / A=1.4$, show that the elongation versus $\chi$ characteristics reproduces well the experimental values at small enough $\chi$.

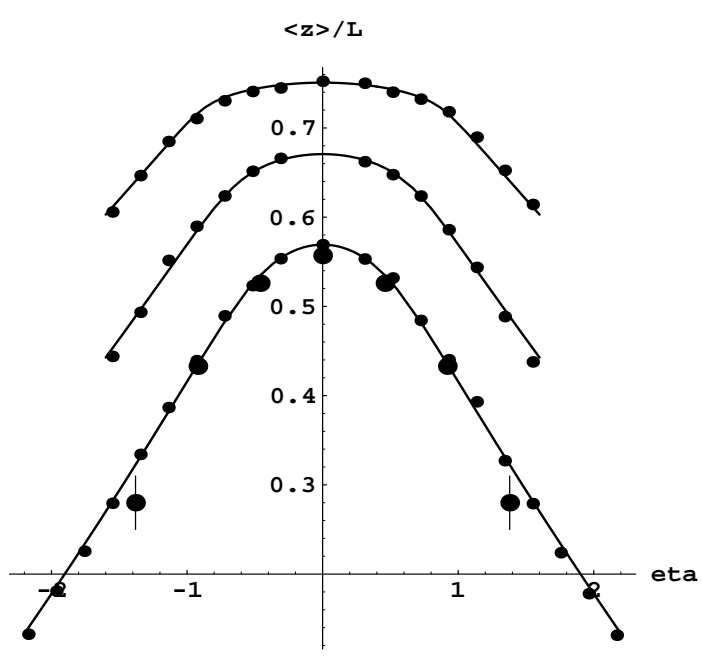

FIG. 1. The elongation versus reduced supercoiling angle $\eta=\chi A / L$ for forces $F=.116, .197, .328 p N$, from bottom to top. $\chi$ is taken directly from experiment and $\eta$ has been computed with $A=51.35 \mathrm{~nm}$ and $L=15.61 \mu \mathrm{m}$. The experimentalist's $\sigma$ is related to $\eta$ by $\eta=\omega_{0} A \sigma=2 \pi \frac{A}{p}=94.8 \sigma$. The smaller points are the experimental results, the bigger points on the lowest curve are from Monte Carlo simulations, the full lines is the analytic study of the WLRC through the parametric representation (9) with the values $C / A$ given in the text.

The regularization in the discrete model comes from the fact that the angle $\phi-\phi^{\prime}$ is defined modulo $2 \pi$. The computation of $\left\langle\chi_{W}^{2}\right\rangle$ can be done explicitely at $\chi=0$, and one finds that the continuum expression $L / A\langle(1-\cos \theta) /(1+\cos \theta)\rangle$ must be substituted by $L / A\left\langle(1-\cos \theta) /(1+\cos \theta) R\left(\sin ^{2} \theta A / b\right)\right\rangle$, where the regularization function is given in terms of Bessel functions by: $R(x)=I_{1}(x) / I_{0}(x)$. It is reasonable to assume that the discrete model is well approximated by the Hamiltonian $\hat{H}^{r}$ which is obtained from $\hat{H}$ by this same substitution. We have computed the elongation properties of the WLRC from the ground state energy of the corresponding regularized rod hamiltonian $\hat{H}^{r}$. The variations of $\frac{\langle z\rangle}{L}$ with $\chi$ now scale as a function of $\chi / L$, as in experiments. We introduce the intensive linking variable $\eta=\chi A / L$ (related to the experimentalists' $\sigma$ by $\left.\sigma=\eta / \omega_{0} A\right)$. The partition function in (7) can be computed by the saddle point method in the limit $L / A \gg 1$ with $\eta$ kept fixed. The saddle point is imaginary, $k=i \kappa(\alpha)$, and from its value one easily deduces the elongation of the chain using the general formulas given above. We obtain in this way the following parametric representation of the bell shape curves giving $\frac{\langle z\rangle}{L}$ versus $\eta$, for a fixed value of the force $\alpha$. 


$$
\frac{A}{C}+2 \frac{\partial \epsilon_{0}}{\partial k^{2}}\left(\alpha,-\kappa^{2}\right)=\frac{\eta}{\kappa} ; \frac{\langle z\rangle}{L}=-\frac{\partial \epsilon_{0}}{\partial \alpha}\left(\alpha,-\kappa^{2}\right)
$$

An alternative to solving the wave equation associated with the regularized hamiltonian $\hat{H}^{r}$ is to work directly with the discretized version of the effective energy (4), using standard transfer matrix methods. We have checked that these two procedures give results for $\epsilon_{0}\left(\alpha,-\kappa^{2}\right)$ which are in very good agreement. Using interpolation techniques, one can eliminate $\kappa$ in $(9)$ in order to obtain the ratio $C / A$ in terms of $\eta,\langle z\rangle / L$ and $b / A$. For various values of the force and of $b / A$, we have computed the empirical value of $C / A$ obtained from each experimental point $\eta,\langle z\rangle / L$. With $b / A=.12$ all these values cluster nicely, allowing for a rather precise determination of $C / A$. For three values of the force, $F=.116 p N ; .197 p N ; .328 p N$, we find respectively $C / A=1.67 \pm .12 ; 1.66 \pm .10 ; 1.71 \pm .09$ (in this analysis we have restricted to the range of small enough supercoiling: $|\eta| \leq 1.5$ for $F=.197, .328 p N$ and $|\eta| \leq 2.2$ for $F=.116 p N)$. For each force, the value of $C / A$ is the result of a statistical weighted average and the quoted error is just the root mean square deviation, read off directly from the data. These three results agree and indicate a value of $C / A \simeq 1.68$. However it should be stressed that the experimental points used here are preliminary and the study of systematic errors in the experiment is not yet completed so this value of $C / A$ is not yet definitive. The bell shape curves, computed for each force with the corresponding value of $C / A$, are compared to the experimental points in fig1. The agreement looks very satisfactory, indicating an overal consistency of the procedure. We have performed the same analysis with $b / A=.05, .2$. With $b / A=.2$ the agreement remains fairly good, but the value $C / A$ for the three values of the forces exhibit fluctuations around 1.68 which can reach $20 \%$. The value $b / A=.05$ appears to be excluded.

Another possible method to get the ratio $C / A$ could be to measure the curvature of the bell shape curve , $\Gamma_{\eta}=\partial^{2}(\langle z\rangle / L) / \partial \eta^{2}$, at its maximum, which can be related to $a_{1}(\alpha)=2\left(\frac{\partial}{\partial k^{2}} \epsilon_{0}\left(k^{2}, \alpha\right)\right)_{k=0}$ through perturbation theory. The $A / C$ is given in terms of the known function $a_{1}(\alpha)$ [14] as:

$$
\frac{A}{C}=-a_{1}(\alpha)+\sqrt{\frac{\partial a_{1} / \partial \alpha}{\Gamma_{\eta}}}
$$

At the moment the measurements of $\Gamma_{\eta}$ is however not precise enough for a good determination with this method.

We have shown that the WLRC must be regularised at small length scale. The corresponding model can be solved analytically and it accounts well for experimental results at small supercoiling, giving a good method to determine the elastic constants ratio $C / A$. Obviously our theory is limited to the small force - small supercoiling regime. For instance the experiments show that for
$F>.45 p N$ the extension is not symmetric for $\chi \rightarrow-\chi$. This kind of effect is totally beyond our simple elastic model which is intrinsically symmetric. Extending it requires the introduction of self avoidance to treat properly the plectoneme formation. The self avoidance will also naturally provide a cutoff length scale. However taking it into account properly is a major challenge 16.

We wish to thank J.-F. Allemand, D. Bensimon, V. Croquette and T.R. Strick for numerous exchanges, as well as J.-P. Bouchaud, A. Comtet and C. Monthus for useful discussions on the winding distribution of random walks. While completing this work, we learned that $\mathrm{P}$. Nelson and his collaborators are working on the same problem in the limit of large forces.

[1] Unité propre du CNRS, associée à l'Ecole Normale Supérieure et à l'Université de Paris Sud

[2] S.B. Smith, L. Finzi and C. Bustamante, Science 258, 1122 (1992).

[3] C. Bustamante, J.F. Marko, E.D. Siggia and S. Smith, Science 265, 1599 (1994).

[4] M. Fixman and J. Kovac, J. Chem. Phys. 58, 1564 (1973).

[5] T.R. Strick, J.-F. Allemand, D. Bensimon, A. Bensimon and V. Croquette, Science 271, 1835 (1996).

[6] J.H. White, Amer. J. Math. 91, 693 (1969); F.B. Fuller, Proc. Nat. Acad. Sci. USA 68, 815 (1971)

[7] T.C. Boles, J.H. White and N.R. Cozzarelli, J. Mol. Biol. 213, 931 (1990).

[8] B. Fain, J. Rudnick and S. Östlund, cond-mat/9610126.

[9] J.F. Marko and E.D. Siggia, Science 265, 506 (1994); Phys. Rev. E52, 2912 (1995).

[10] See the discussion in A. Comtet, J. Desbois and C. Monthus, J. Stat. Phys. 73, 433 (1993), and references therein, particularly: F. Spizer, Trans. Am; Math. Soc. 87, 187 (1958); C. Belisle, Ann. Prob. 17, 1377 (1989).

[11] The quantity $2 \pi \chi_{W}$ reproduces the writhe of a closed chain only modulo $4 \pi \sqrt[8]{8}$, but it has the advantage of being the integral of a local quantity.

[12] See A.V. Vologodskii, S.D. Levene, K.V. Klenin, M. Frank-Kamenetskii and N.R. Cozzarelli, J. Mol. Biol. 227, 1224 (1992) and references therein.

[13] J.F. Marko and A.V. Vologodskii, Biophys. Journ. 73, 123 (1997).

[14] C. Bouchiat and M. Mézard, in preparation.

[15] J.-F. Allemand, D. Bensimon, V. Croquette and T.R. Strick, in preparation.

[16] A first attempt can be found in: J.D. Moroz and R.D. Kamien, 'Self avoiding walks with Writhe', condmat/9705066 